In this paper, the study of the dynamical behavior of logistic map has been disused with representing fractals graphics of map, the logistic map depends on two parameters and works in the complex plane, the map defined by $f(z, \alpha, \beta)=\alpha z(1-z) \beta$, where $z$ and $\alpha$ are complex numbers, and $\beta$ is a positive integers number, the visualization method used in this work to generate fractals of the map and to inspect the relation between the value of $\beta$ and the shape of the map, this visualization analysis showed also that, as the value of $\beta$ increasing, as the number of humps in the function also increasing, and it demonstrate that is true also for the function's first iteration, $f^{2}\left(x_{0}\right)=f\left(f\left(x_{0}\right)\right)$ and the second iteration, $f^{3}\left(x_{0}\right)=f\left(f^{2}\left(x_{0}\right)\right)$, beside that, the visualization technique showed that the number of humps in that fractal is less than the ones in the second iteration of the original function, the study of the critical points and their properties of the logistic map also discussed it, whereas finding the fixed point led to find the critical point of the function $f$, in addition, it haven proven for the set of all points $\alpha \in C$ and $\beta \in N$, the iteration function $f(f(z)$ has an attractive fixed points, and belongs to the region specified by the disc $|1-\beta(\alpha-1)|<1$. Also, The discussion of the Mandelbrot set of the function defined by the $f(f(z))$ examined in complex plans using the path principle, such that the path of the critical point $z=z_{0}$ is restricted, finally, it has proven that the Mandelbrot set $f(z, \alpha, \beta)$ contains all the attractive fixed points and all the complex numbers $\alpha$ in which $\alpha \leq(1 / \beta+1)(1 / \beta+1)$ and the region containing the attractive fixed points for $f^{2}(z, \alpha, \beta)$ was identified

Keywords: fixed points; logistic map; quadratic map; Mandelbrot set; Zoomer Xaos
UDC 546

DOI: $10.15587 / 1729-4061.2021 .249264$

\section{DEVELOPMENT OF MANDELBROT SET FOR THE LOGISTIC MAP WITH TWO PARAMETERS IN THE COMPLEX PLANE}

\author{
Wasan Saad Ahmed \\ Master in Computer Science* \\ Sa ad Qasim Abbas \\ Doctor of Mathematics, Professor \\ Department of Medical Instrumentation of Technology \\ Bilad Alrafidain University College \\ Diyala Junction, Diyala, Iraq \\ Muntadher Khamees \\ Doctor in Computer Science, Professor* \\ Mustafa Musa Jaber \\ Corresponding author \\ $\mathrm{PhD}$, Lecturer \\ Department of Medical Instrumentation Techniques Engineering \\ Dijlah University College \\ AlMasafi str., Baghdad, Iraq, 00964 \\ Department of Computer Technology Engineering \\ AL-Turath University College \\ Almansor, Baghdad, Iraq \\ E-mail: mustafa.musa@duc.edu.iq \\ *Department of Computer Science \\ University of Diyala \\ Baqubah, Diyala, Iraq
}

Received date 29.10.2021

Accepted date 14.12.2021

Published date 29.12.2021
How to Cite: Ahmed, W. S., Abbas, S. Q., Khamees, M.,Jaber, M. M. (2021). Development of Mandelbrot set for the logistic map with two parameters in the complex plane. Eastern-European Journal of Enterprise Technologies, 6 (3 (114)), 47-56. doi: https://doi.org/10.15587/1729-4061.2021.249264

\section{Introduction}

Many researchers studied the Mandelbrot set in detail because of the importance of the complex plan [1-3], in editions to that, the accomplishment of this set as a new approach in the extraordinary complex set $[4,5]$. The application of Mandelbrot can be found in a variety of fields such as coat line sample creation, terrain navigation of low altitude flying, road potential placement, bridges, tunnels, and other area management, transportation, and management [6]

The logistic map (sometimes considered as equivalently, recurrence relation [7] is an equation of the form $\alpha z(1-z)^{\beta}$, where $\alpha$ is a real number and $\beta$ is a non-zero number. The logistic map is a quadratic polynomial from the recurrence relation point of view, and a considered a good representation of the unexpected and difficult behavior of basic dynamic non-linear equations. In 1975 biologist Robert May [8] was the first person who introduces this map in his work, in 1976 May gives a more detailed introduction about this map in [4]

$$
\alpha z(1-z)^{\beta} M=\left\{c \in \mathbb{C}, f_{c}^{k}(0) \nrightarrow \infty \text { as } k \rightarrow \infty\right\} c, z \in \mathbb{C}
$$

Let's note that the Mandelbrot set has the initial value when $z=0$. Many researchers studied this set in detail because of the importance of the complex plan, [9] in editions to that, the accomplishment of this set as a new approach in the extraordinary complex set. Despite the studying of this set being considered as closed from in mathematical point of view $[10,11]$ it is still important to share knowledge among the researchers' society.

Chaos and nonlinear dynamics concepts are considered highly important in engineering and mathematics as well as physics. It is fairly said that linear systems nowadays can be built completed theorem. This theorem is easy to understand and study. Oppose to linear, which involve closed-form solution, the most abstract and complicated phenomenon is nonlinearity [12].

Because of the nature of the quadratic map, it is not possible to construct a symbolic dynamical system unless the map has an inverse; unfortunately, there is no symbolic dynamical sequence since the Mandelbrot set is non-invertible In $[13,14]$ the works of hyperbolic components ordered have explained without finding the symbolic sequences, i.e., 
one cannot know more than the order of the Misiurewicz points and the hyperbolic components of the Mandelbrot set whatever it has been studied deeply.

In recent research's work $[13,15]$ the representative of the chaos paradigm was the Mandelbrot set, and by following Devaney's ideas [16], chaotic function $f$ can be defined as Let $V$ be any set. The function $f: V \mapsto V$ is said to be chaotic on $V$ if the following conditions are satisfied:

1. The function $\mathrm{f}$ is sensitive to the initial conditions.

2 . There is a non-negative integer $\mathrm{n}$ in any non-empty set $U$ and $W$ in $V$ such that $f^{\wedge} n(U) \cap V \neq \varnothing$.

3. The periodic points of the function $f$ are dense in the set $V$.

Despite the extreme complexity of the chaos we are dealing with, most of the time it is not random, because it appropriates to a mathematical formula; hence this chaos is deterministic.

The chaos of any phenomenon (physical or not) can be reached in more than one different direction, the Myrberg-Feigenbaum direction, the intermittence direction, and the quasiperiodic direction [17, 18]. In this work the Myrberg-Feigenbaum direction will be considered, the reason behind that is the existence of this kind of direction in both, one dimension and complex, quadratic maps, besides that the Mandelbrot set is a good reparative of the complex quadratic map. For the one-direction quadratic maps, the direction is simple and has a strong order known as the period-doubling cascade [19, 20].

The real Mandelbrot function $x_{n+1}=x_{n}^{2}+c$ can be used to study the one-dimensional quadratic function, and this idea is similar to using the Mandelbrot set to study the complex case, this real Mandelbrot function represents the intersection point of the one-dimensional real number line with the Mandelbrot set, the value of the parameter $c$ lies between -2 and 0.25 . The multiplier of the real Mandelbrot function has many different forms of points in orbit $\lambda=\left|d\left(f_{c}(x)\right) / d x\right|_{x=x_{f p}}$ of the fixed point, $x_{f p}$.[21] used this definition, in [22] the same miner defines complex map, but $c$ was defined for a wider range of values.

\section{Literature review and problem statement}

Many researchers studied the Mandelbrot set in detail because of the importance of the complex plan [2], in addition to that, the accomplishment of this set as a new approach in the extraordinary complex set. Despite the studying of this set being considered as closed from in mathematical point of view [23] it is still important to share knowledge among the researchers' society.

Because of the nature of the quadratic map, it is not possible to construct a symbolic dynamical system unless the map has an inverse; unfortunately, there is no symbolic dynamical sequence since the Mandelbrot set is non-invertible. Hao and Zheng explained how ordering the hyperbolic components works without finding the symbolic sequences [24], i.e., one cannot know more than the order of the Misiurewicz points and the hyperbolic components of the Mandelbrot set whatever it has been studied deeply. The application of fractal geometry can be noticed palpably in the software engineering field. The first work of fractal appeared in Mandelbrot's paper in 1970, where the visualization of the function $f$ $(z)=z^{2}+c$ is represented in the complex graph [13]. The graph of the function is longitudinally symmetrical. He called it fractions. His work is a compliment and development to the Julia set in [18] it was found through the research that the Julia set is very distinguished in artistic representation.

The Work of Mandelbrot has been developed and different types of fractals have been created, in [14] for example, different types of the generalized Mandelbrot sets were studied. In $[25,26]$ logarithmic, trigonometric, and logical functions were used also, as well as exponential functions to generate different kinds of fractals. In addition to that, it has been used of quadrilateral functions with the bi-compound in [16] as well as the tripartite complexes to generate fractals in [19].

The topic became more important when the fixed point theory was studied by Rani et al. In [25], they used fixed iterative techniques with fixed point property to work for representative and generating fractals. The fractals with advanced and superior properties were produced and their properties were checked and tested as well. With all this effort made by the authors, the fixed point theory turned out to be a typical part of software engineering and mathematics science.

Thus, because of the difficulty in the analysis of dynamical behavior of logistic map parameters, especially when the value of power $\beta$ became very large [27], or when the iterative techniques used to find the composition of the logistic map [28], However, all the previous approaches suffer from complexity, sensitivity to any parameter changing, Therefore, using these methods to analyse the behavioral of Mandelbrot maps made it hard to study due to long complex derivatives and onerous calculations, Therefore, graphical-based methods are used in this work, to study the one-dimensional quadratic function without going through these difficulties. Moreover, the graphical analysis used to show there is a relation between the power of the function $\beta$ and the number of humps in the function, and that is true also for the function's iteration. Also, let's prove that the logistic map has, non-zero attractive fixed points, and belong to the region specified by the disc $|1-\beta(\alpha-1)|<1$, the definition of the Mandelbrot set given and the position of the attractive point proven to be inside the Mandelbrot set and $|\alpha| \leq\left((\beta+1)^{\left(\frac{1}{\beta}+1\right)}\right) / \beta$.

\section{The aim and objectives of the study}

The study aims to analyse the behavior of the logistic map defined by $f(z, \alpha, \beta)=\alpha z(1-z)^{\beta}$.

To achieve this goal the following objectives are accomplished:

- using the graphical technique to show the relation between the power of the function $\beta$ and the number of humps in the function, and demonstrate the same relation for function's iteration;

- the theoretical methods have been used show that the function $f(z, \alpha, \beta)$ defined by $f(z, \alpha, \beta)$ has fixed points, attractive, non-zero and belong to the disc $\mid 1-\beta(\alpha-1 \mid<1$;

- using the theoretical methods it is aimed to prove that function $f(z, \alpha, \beta)=\alpha z\left(1-z^{\wedge \beta}\right)$, where $\alpha \in \mathbb{C}$ and $\beta \neq 0 \in \mathbb{N}$, have an attractive point which belongs to set

$$
M=\left\{\alpha \in \mathbb{C} \text { and } \beta \in \mathbb{N}: O\left(Z_{0}\right) \text { is boubded under } f(z, \alpha, \beta)\right\},
$$

and this Set contains all complex number $\alpha$ in which $|\alpha| \leq(\beta+1)^{\left(\frac{1}{\beta}+1\right)} / \beta ;$ 
- theoretical methods have been used to prove that the set of all points $\alpha \in C$ such that the function $f^{2}(z, \alpha)$ has fixed points attractive and non-zero and belongs to the bounded region.

\section{Materials and methods}

The behavior of the logistic map $f(z, \alpha, \beta)=\alpha z(1-z)^{\beta}$ have been investigated and explained, using the graphical technique and the theoretical methods, the following section explains the process used to achieve this goal. Basic definitions and principles highlighted in which they used in to have the results in this paper.

Definition 1 [29]. A one-dimensional dynamical system is a pair $(I, f)$ where $f$ is a function $f: I \rightarrow I$, and $I$ is a subset of $R$ nearly permanently, the interval $I$ will be a subinterval of $R$, that contains the probability $I=R$.

Definition 2 [30]. Given $x_{0} \in R$ the orbit of $x_{0}$ under $f$ is the set where

$$
O\left(x_{0}\right)=\left\{x_{0}, f\left(x_{0}\right), f^{2}\left(x_{0}\right), f^{3}\left(x_{0}\right), \ldots\right\},
$$

where

$$
\begin{aligned}
& f^{2}\left(x_{0}\right)=f\left(f\left(x_{0}\right)\right) \\
& f^{3}\left(x_{0}\right)=f\left(f^{2}\left(x_{0}\right)\right) \\
& f^{n}\left(x_{0}\right)=f \circ f_{0} f \circ \ldots f\left(x_{0}\right) \text { (n-times composition). }
\end{aligned}
$$

For each $n \in \mathbb{N}$. .

Definitions 3 [31]. A point $x^{*}$ is said to be a fixed point of the map $f$ or equilibrium point if $f(x)=x^{*}$.

Theorem 1 [32]: Suppose that if is differentiable at a fixed point $x$.

If $|f(x)|<1$, then $x$ is attractive (stable).

If $|f(x)|>1$, then $x$ is repelling (unstable).

If $|f(x)|=1$, then $x$ is attractive repelling or neither.

To analyse the dynamic behavior of the logistic function The visualization methods have been used, specifically, the real-time fractal Zoomer Xaos software used to represent the fractals graphics of the logistic function [33], the values of $\alpha$ taken randomly, while the value of $\beta$ lies between 1 and 30, when $\beta$ under 10 the graph will seem like a maple leaf, but when $\beta$ exceeded 10 there is what is look like humps (Fig. 1) appears on the outside cover of the leaf.

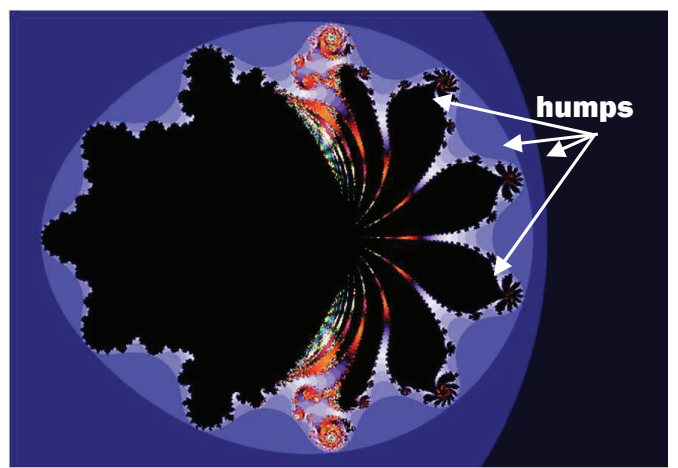

Fig. 1. The humps of the logistic function $f(z)=\alpha z(1-z)^{\beta}, \beta<10$

When the value of $\beta$ grows up the number of humps also increases. In addition, the number of humps is directly proportional to the value of $\beta$, and these numbers are always less than or equal to the power of $(1-z)^{\beta}$. Fig. 2 showed the fractals of $f(z)$ for different values of $\beta$.

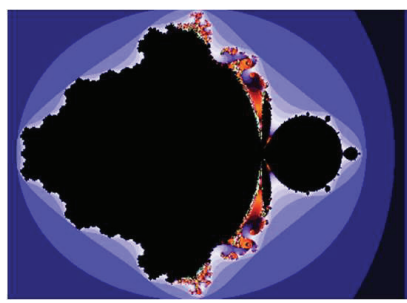

$a$

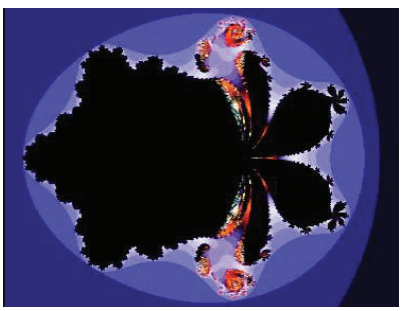

C

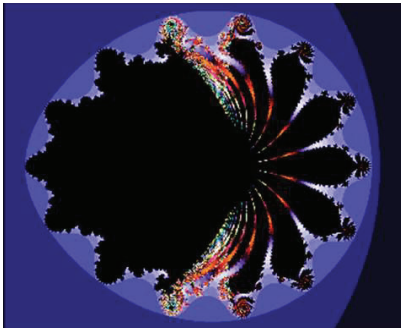

e

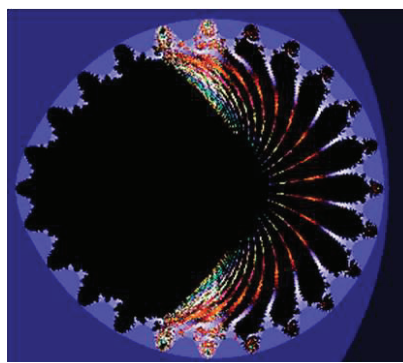

g

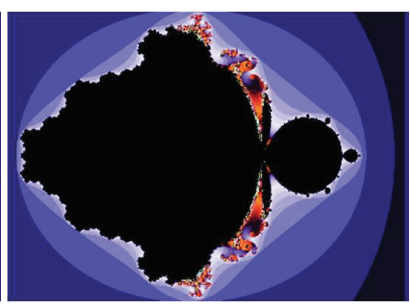

b

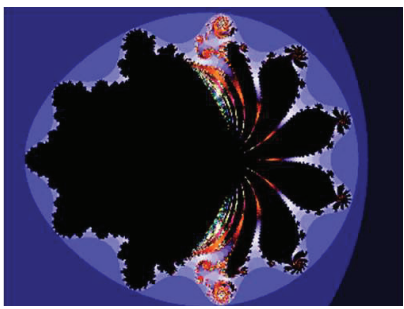

d

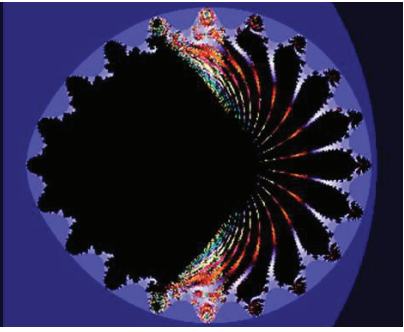

$f$

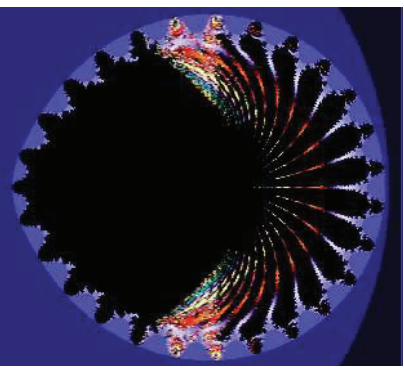

h

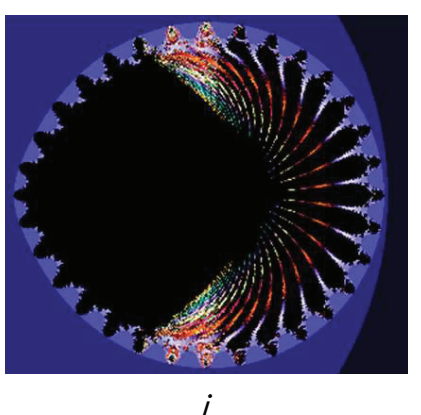

Fig. 2. Graphical representation of $f(z)=\alpha z(1-z)^{\beta}$ for different values of $\beta$ : $a-\beta=2 ; b-\beta=3 ; c-\beta=5 ; d-\beta=9$; $e-\beta=12 ; f-\beta=18 ; g-\beta=22 ; h-\beta=25 ; i-\beta=30$

Obviously from Fig. 2, the number of humps in the graph increases as the power of $(1-z)$ i.e. the $\beta$, increases.

Remark1. The number of humps in the fractals of function $\alpha z(1-z)^{\beta}$ always less than or equal to $\beta$. 
It is very clear from Fig. 2 the above remark is true for the logistic function $\alpha z(1-z)^{\beta}$.

In the same way, wit is possible to represent the fractal of the logistic function iteration, the value of the $\beta$ taken so that the software can achieve the graph, for this reason, the beta value lies between 1 and 2 i.e.

\section{$1 \leq \beta \leq 2$.}

For $\beta=1$, the first iteration will be

$$
\begin{aligned}
& f(f(z))=c\left(c z(1-z)^{1}\right)\left(1-\left(c^{*} z^{*}(1-z)^{1}\right)\right)^{1}= \\
& =\left(-c^{3}\right) z^{4}+\left(2^{*} c^{3}\right) z^{3}+\left(-c^{3}-c^{2}\right) z^{2}+c^{2} z .
\end{aligned}
$$

The fractal of the first iteration shows that the number of humps is less than the ones in the second iteration

$$
\begin{aligned}
& f(f(f(z)))=c^{*}\left(\begin{array}{l}
-c^{3 *} z^{4}+2 * c^{3 *} z^{3}- \\
-c^{3 *} z^{2}-c^{2 *} z^{2}+c^{2 *} z
\end{array}\right) \times \\
& \times\left(1\left(\begin{array}{l}
-c^{3 *} z^{4}+2 * c^{3 *} z^{3}- \\
-c^{3} z^{2}-c^{2} * z^{2}+c^{2 *} z
\end{array}\right)\right)^{1} .
\end{aligned}
$$

Fig. 2, $a, b$ represents (1) and (2) respectively, it is clear that the last ( $b$ ) one is more rounded, while Fig. 3, $c, d$ below, represent the same equations but with applying a $3 \mathrm{~d}$ fitter on them.

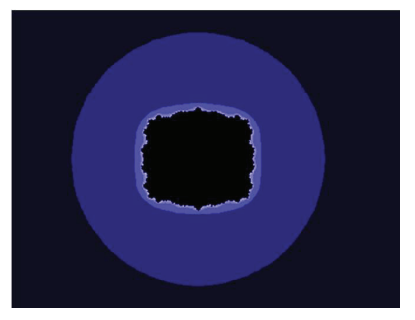

$a$

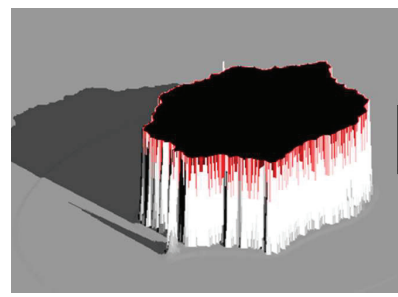

c

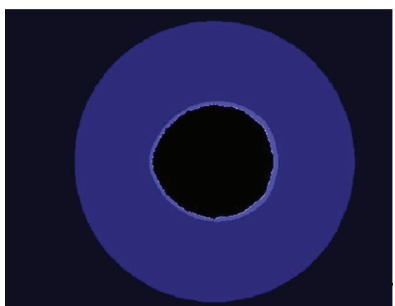

$b$

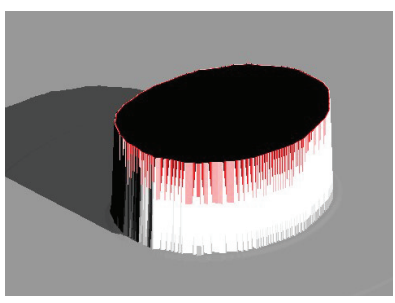

$d$
Fig. 3. Graphical representation of fractal: $a-\beta=1, f(f(z))$; $b-\beta=1, f(f(f(z))) ; c-\beta=1, f(f(z))$ with 3d fitter; $d-\beta=1$, $f(f(f(z)))$ with $3 d$ fitter

To demonstrate, the relationship between the fixed point and the critical point of the function $f(z, \alpha, \beta)$ and to determine when the fixed point is attractive, we need as a start point, the fixed-point concepts, as follow, considering the function

$$
f(z, \alpha, \beta)=\alpha z(1-z)^{\beta},
$$

where $z$ and $\alpha \in C, \beta \in N$, The function $f(z, \alpha, \beta)$ is analytic and differentiable, the roots of $f^{\prime}(z, \alpha, \beta)=0$ is the same as the critical points of the function in (3), to find the fixed point first it is necessary to find the critical point of the function $f$, according to that

$$
\begin{aligned}
& f(z, \alpha, \beta)=\alpha-(\beta+1) \alpha \cdot z^{\beta}=0, \\
& (\beta+1) z^{\beta}=1, \\
& z^{\beta}=\frac{1}{(\beta+1)}, \\
& z=\left(\frac{1}{\beta+1}\right)^{\frac{1}{\beta}},
\end{aligned}
$$

which is the critical point of $f(z, \alpha, \beta)$ while the Fixed points of the function $f(z, \alpha, \beta)=z$

$$
\begin{aligned}
& \alpha z-\alpha z^{\beta+1}-z=0, \\
& z\left(\alpha-\alpha z^{\beta}-1\right)=0 .
\end{aligned}
$$

Either $z=0$ or $\alpha z^{\beta}=\alpha-1$

$$
\begin{aligned}
& z^{\beta}=\alpha-1, \\
& z^{\beta}=\frac{\alpha-1}{\alpha}, \\
& z=\left(\frac{\alpha-1}{\alpha}\right)^{1 / \beta} .
\end{aligned}
$$

So the fixed points for the function $f(z, \alpha, \beta)$ are $z=0$ and $z((\alpha-1) / \alpha)^{1 / \beta}$.

And to prove it is attractive it should be achieved in the following way, explained below

$$
\begin{aligned}
& \left|f^{\prime}(z, \alpha, \beta)\right|<1, \\
& \left|f^{\prime}\left(\left(\frac{\alpha-1}{\alpha}\right)^{1 / \beta}, \alpha, \beta\right)\right|<1 .
\end{aligned}
$$

Form (2)

$$
f^{\prime}(z, \alpha, \beta)=z-\alpha(\beta+1) z^{\beta} .
$$

To make fixed points in the equation (6), let's obtain

$$
\begin{aligned}
& f^{\prime}(0, \alpha, \beta)=\alpha, \\
& f^{\prime}\left(\left(\frac{\alpha-1}{\alpha}\right)^{1 / \beta}, \alpha, \beta\right)=z-(\alpha-1)(\beta+1) .
\end{aligned}
$$

And applying (4) and (5), there is

$$
|\alpha|<1, \quad|1-\beta(\alpha-1)|<1 .
$$

Theorem 2. The set of all points $\alpha \in \mathbb{C}$ and $\beta \in \mathbb{N}$, such that the function $f(z, \alpha, \beta)$ defined by the eq. (1) has fixed points attractive and non-zero, belong to the region specified by the disc $\mid 1-\beta(\alpha-1 \mid<1$.

Proof.

Let $f(z, \alpha, \beta)$ be the function defined in the (1), the non-zero fixed points of the function $f(z, \alpha, \beta)$ is:

$$
z=\left(\frac{\alpha-1}{\alpha}\right)^{1 / \beta}, \alpha \in \mathbb{C} \text { and } \beta \neq 0 \in \mathbb{N}
$$


and be attractive if

$$
\left|f^{-1}\left(\left(\frac{\alpha-1}{\alpha}\right)^{1 / \beta}, \alpha, \beta\right)\right|=|1-\beta(\alpha-1)|<1 .
$$

Let $w$ complex number and suppose that

$$
\begin{aligned}
& w=\beta(\alpha-1), \\
& |1-w|<1 .
\end{aligned}
$$

In a complex plan the function $|1-w|$ considered a circle that has a radius one and center $(1,0)$ and passes in the origin point. it is expressed by polar coordinate as $r=2 \cos \theta$, and shown in Fig. 4, $a$.

But when $w=\beta \alpha-\beta$ then

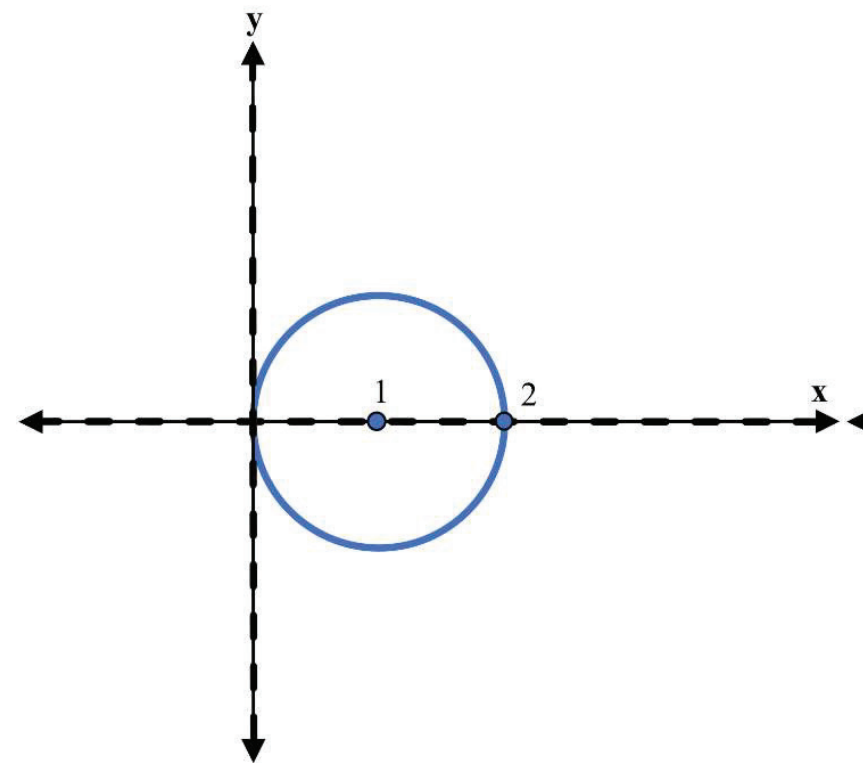

$a$

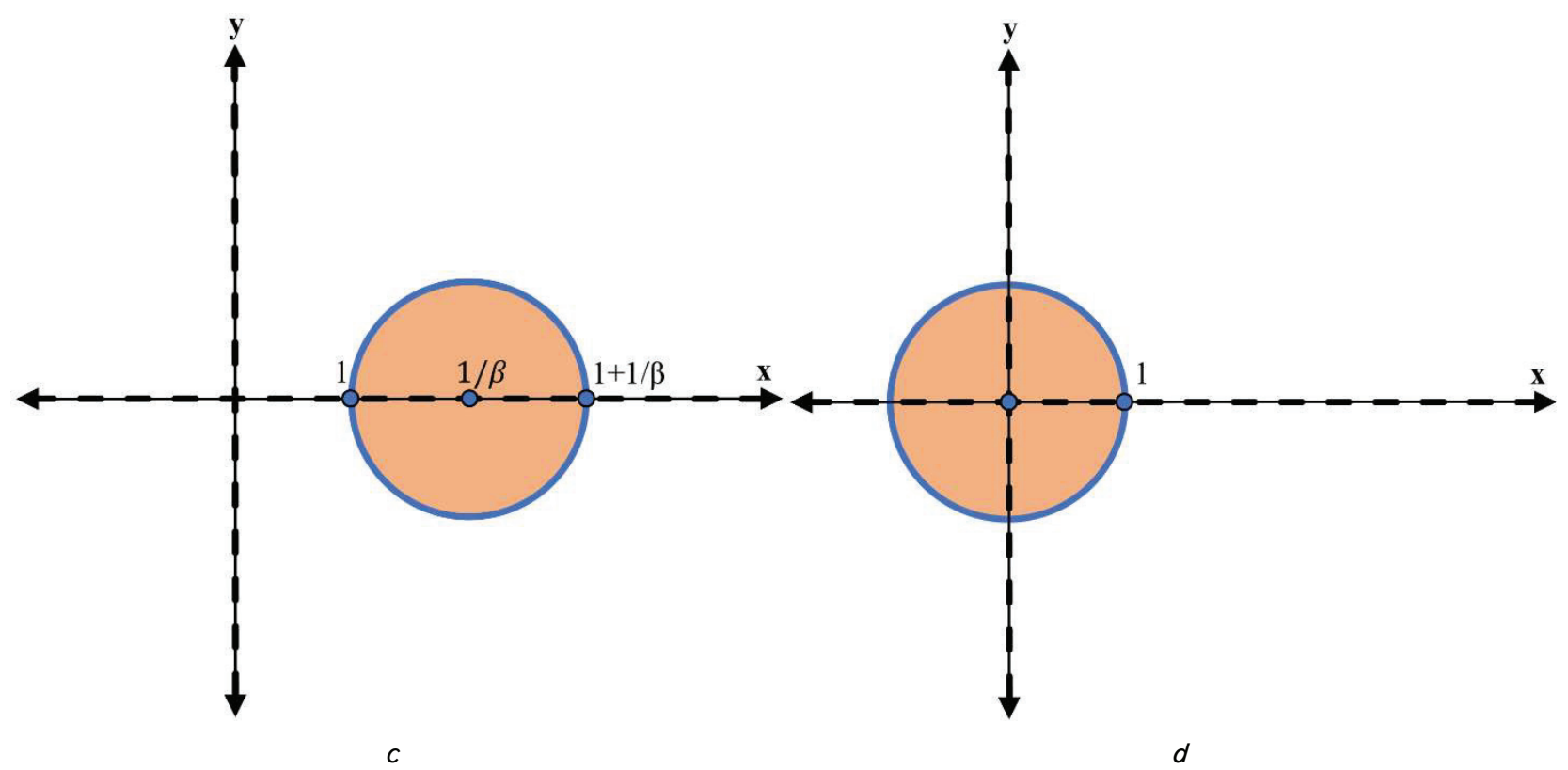

Fig. 4. The representation in the complex plan of the function $|1-w|=r . a-|1-w|=1 ; b-|1-\beta(\alpha-1)|=\frac{1}{\beta}$;
\[ c-|1-\beta(\alpha-1)|<1 ; d-|w|=1 \]
That means, the value $\alpha$ represents the circumference of the circle cantered $\left[\left(\frac{1}{\beta}+1\right), 0\right]$ and radius $\frac{1}{\beta}$, as shown in Fig. 4, $b$. Finally, $|1-\beta(\alpha-1)|<1$ in complex plan represent all values of $\alpha \in \mathbb{C}$ and the non-zero $\beta \in \mathbb{N}$, which represent the disk shown in Fig. 4, $c$.

Remark 2. The fixed point $z=0$ is an attractive point, and that its path is the origin point, As shown in Fig. 4, $d$.

The discussion of the Mandelbrot set of the function $f(z, \alpha, \beta)$ defined by (3) using the path principle as follow.

Definition 3. Let $M$ is the Mandelbrot set of the function $(z, \alpha, \beta)$, it is defined as a set of all complex number $\alpha \in \mathbb{C}$ and non-zero $\beta \in \mathbb{N}$, such that the path of the critical point $z=z_{0}$ is restricted and the set $M$ as follows:

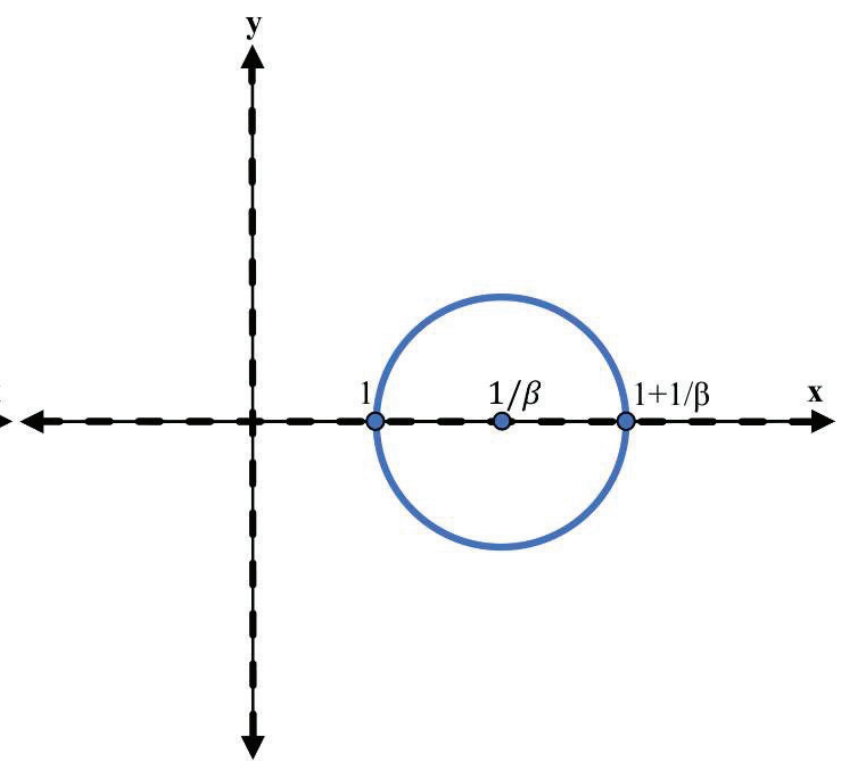

$b$

$$
c-|1-\beta(\alpha-1)|<1 ; d-|w|=1
$$




$$
M=\left\{\begin{array}{l}
\alpha \in \mathbb{C} \text { and } \beta \in \mathbb{N}: O\left(Z_{0}\right) \\
\text { is boubded under } f(z, \alpha, \beta)
\end{array}\right\},
$$

where $O\left(Z_{0}\right)$ is symbolizes the path of the critical point.

Theorem 3. Let $M$ be the Mandelbrot set for the function $f(z, \alpha, \beta)=\alpha z\left(1-z^{\wedge} \beta\right), \alpha \in \mathbb{C}$ and $\beta \neq 0 \in \mathbb{N}$, then:

1. The Points of attractive for $f(z, \alpha, \beta)$ belong to $M$.

2 . The Set $M$ contains all complex number $\alpha$ in which $|\alpha| \leq(\beta+1)^{\left(\frac{1}{\beta}+1\right)} / \beta$.

Proof.

Since

$$
f^{-1}(z, \alpha, \beta)=\alpha-\alpha(\beta+1) z^{\beta},
$$

so

$$
f^{-1}\left(\left(\frac{\alpha-1}{\alpha}\right)^{1 / \beta}, \alpha, \beta\right)=0
$$

Then the function $f(z, \alpha, \beta)$ has critical points when $z=\left(\frac{1}{\beta+1}\right)^{1 / \beta}$.

The function $f(z, \alpha, \beta)$ has attractive points when

$$
z=0,|\alpha|<1 \mid
$$

and

$$
z=\left(\frac{\alpha-1}{\alpha}\right)^{1 / \beta}, \mid 1-\beta(\alpha-1 \mid<1 .
$$

Then the critical points belong to the basin of attraction, and there exist integer number $n \in \mathbb{Z}^{+}$, so that

$$
\lim _{n \rightarrow \infty} f^{n}\left(\left(\frac{1}{\beta+1}\right)^{1 / \beta}, \alpha, \beta\right)=\left(\frac{\alpha-1}{\alpha}\right)^{1 / \beta} \Rightarrow O\left(\frac{1}{\beta+1}\right)^{1 / \beta}
$$

are bounded

$$
\begin{aligned}
& \Rightarrow\left(\frac{\alpha-1}{\alpha}\right)^{1 / \beta} \in M \\
& \text { Let }|\alpha| \leq\left(\frac{1}{\beta+1}\right)^{\frac{1}{\beta}+1}, \\
& \left.\left.|f|\left(\frac{1}{\beta+1}\right)^{1 / \beta}, \alpha, \beta\right)|=| \alpha\left(\frac{1}{\beta+1}\right)^{1 / \beta} \mid 1-\left(\left(\frac{1}{\beta+1}\right)^{\frac{1}{\beta}}\right)^{\beta}\right) \mid= \\
& =\left|\alpha\left(\frac{1}{\beta+1}\right)^{\frac{1}{\beta}}\left(1-\frac{1}{\beta+1}\right)\right|=\left|\alpha\left(\frac{1}{\beta+1}\right)^{\frac{1}{\beta}}\left(\frac{\beta}{\beta+1}\right)\right|= \\
& \left.=|\alpha| \frac{\beta}{\left.(\beta+1)^{\left(\frac{1}{\beta}+1\right.}\right)}|\leq| \frac{(\beta+1)\left(\frac{1}{\beta}+1\right)}{\beta}\right)\left|\frac{\beta}{\left.(\beta+1)^{\left(\frac{1}{\beta}+1\right.}\right)}\right|= \\
& =1 \Rightarrow\left|f\left(\left(\frac{1}{\beta+1}\right)^{1 / \beta}, \alpha, \beta\right)\right| \leq 1
\end{aligned}
$$

$$
\begin{aligned}
& f^{2}(z, \alpha, \beta)=f(f(z, \alpha, \beta))= \\
& =\alpha^{2} z\left(1-z^{\beta}\right)\left(1-\alpha^{\alpha} z^{\beta}\left(1-z^{\beta}\right)^{\beta}\right) . \\
& \alpha^{2}\left(\frac{1}{\beta+1}\right)^{1 / \beta}\left(1-\frac{1}{\beta+1}\right) \times \\
& \left|f^{2}\left(\left(\frac{1}{\beta+1}\right)^{1 / \beta}, \alpha, \beta\right)\right|=\left|\times\left(1-\alpha^{\beta}\left(\frac{1}{\beta+1}\right)^{1 / \beta}\right)-\right|= \\
& -\left(1-\left(\frac{1}{\beta+1}\right)^{\beta}\right) \\
& =\left|\alpha^{2}\left(\frac{1}{\beta+1}\right)^{1 / \beta} \frac{\beta}{\beta+1}\left(1-\alpha^{\beta}\left(\frac{1}{\beta+1}\right)^{\beta}\left(\frac{\beta}{\beta+1}\right)^{\beta}\right)\right|= \\
& =\left|\alpha^{2}\right|\left|\frac{\beta}{(\beta+1)^{\frac{1}{\beta}+1}}\left(1-\frac{\alpha^{\beta} \beta^{\beta}}{(\beta+1)^{\beta}}\left(\frac{1}{\beta+1}\right)^{1 / \beta}\right)\right| \leq \\
& \leq|\alpha| \frac{(\beta+1)^{\frac{1}{\beta^{+1}}}}{\beta} \frac{\beta}{(\beta+1)^{\frac{1}{\beta}+1}}\left(1-\left(\frac{\alpha \beta}{\beta+1}\right)^{\beta}\right)\left(\frac{1}{\beta+1}\right)^{1 / \beta} \mid= \\
& =|\alpha|\left(1-\frac{\beta}{(\beta+1)^{\frac{1}{\beta}+1}} \alpha\left(\frac{\alpha \beta}{\beta+1}\right)^{\beta-1}\right) \leq \\
& \leq|\alpha|-\left|\frac{\beta}{(\beta+1)^{\frac{1}{\beta}+1}} \alpha\left(\frac{\alpha \beta}{\beta+1}\right)^{\beta-1}\right| \leq 1 \text {. }
\end{aligned}
$$

Using Mathematical induction, let's assume the relationship is true when $k=n$

$$
\left|f^{n}\left(\left(\frac{1}{\beta+1}\right)^{1 / \beta}, \alpha, \beta\right)\right| \leq 1 .
$$

And now for $k=n+1$

$$
\begin{aligned}
& \left|f^{n+1}\left(\left(\frac{1}{\beta+1}\right)^{\frac{1}{\beta}}, \alpha, \beta\right)\right|=\mid f\left(f^{n}\left(\left(\frac{1}{\beta+1}\right)^{\frac{1}{\beta}}, \alpha, z\right) \mid=\right. \\
& \left.=\left|\alpha f^{n}\left(\left(\frac{1}{\beta+1}\right)^{\frac{1}{\beta}}, \alpha, z\right)\right| 1-\left(f\left(\left(\frac{1}{\beta+1}\right)^{\frac{1}{\beta}}, \alpha, \beta\right)\right)^{\beta}\right) \mid= \\
& \left.=|\alpha| \mid f^{n}\left(\left(\frac{1}{\beta+1}\right)^{\frac{1}{\beta}}, \alpha, \beta\right)\left(1-\left(f\left(\left(\frac{1}{\beta+1}\right)^{\frac{1}{\beta}}, \alpha, \beta\right)\right)\right)^{\beta}\right) \mid \\
& \text { Since }|\alpha| \leq\left(\frac{(\beta+1)^{\frac{1}{\beta}+1}}{\beta}\right) \text { from (2) then } \\
& \left|f^{n+1}\left(\left(\frac{1}{\beta+1}\right)^{\frac{1}{\beta}}, \alpha, \beta\right)\right| \leq 1 .
\end{aligned}
$$


So, the relationship is correct for all $k$, then

$$
\begin{aligned}
& \left|f^{k}\left(\left(\frac{1}{\beta+1}\right)^{\frac{1}{\beta}}, \alpha, \beta\right)\right| \leq 1 \text { for all } k \geq 1, \\
& \Rightarrow O\left(\frac{1}{\beta+1}\right)^{1 / \beta}
\end{aligned}
$$

bounded $\Rightarrow M$ contains all complex numbers $\alpha$ and non-zero $\beta \in \mathbb{N}$, such that $|\alpha| \leq\left(\frac{(\beta+1)^{\frac{1}{\beta}+1}}{\beta}\right)$.

Remark 3. Consider the function $f(z, \alpha)=\alpha z(1-z), \alpha \in \mathbb{C}$, and $\beta=1$, then

$$
\begin{aligned}
& f^{2}(z, \alpha)=\alpha^{2} z(1-z)[1-\alpha z(1-z)]= \\
& =\alpha^{2} z-\alpha^{2}(1+\alpha) z^{2}+z \alpha^{3} z^{2}-\alpha^{3} z^{4}
\end{aligned}
$$

Remark 4. The region that contains the function $f^{2}(z, \alpha)$ can represent in the following graph.

Theorem 4. The set of all points $\alpha \in C$ such that the function $f^{2}(z, \alpha)$ is defined by (8) has fixed points attractive and non-zero and belong to the region specified by shown in Fig. 5 .

In the next theorem, let's prove that the shape Fig. 5 . is the region that contains the function $f^{2}(z, \alpha)$ ).

Proof.

To find the fixed points of a function $f^{2}(z, \alpha)$, it is possible to do the following analysis

$$
f^{2}(z, \alpha)=z
$$

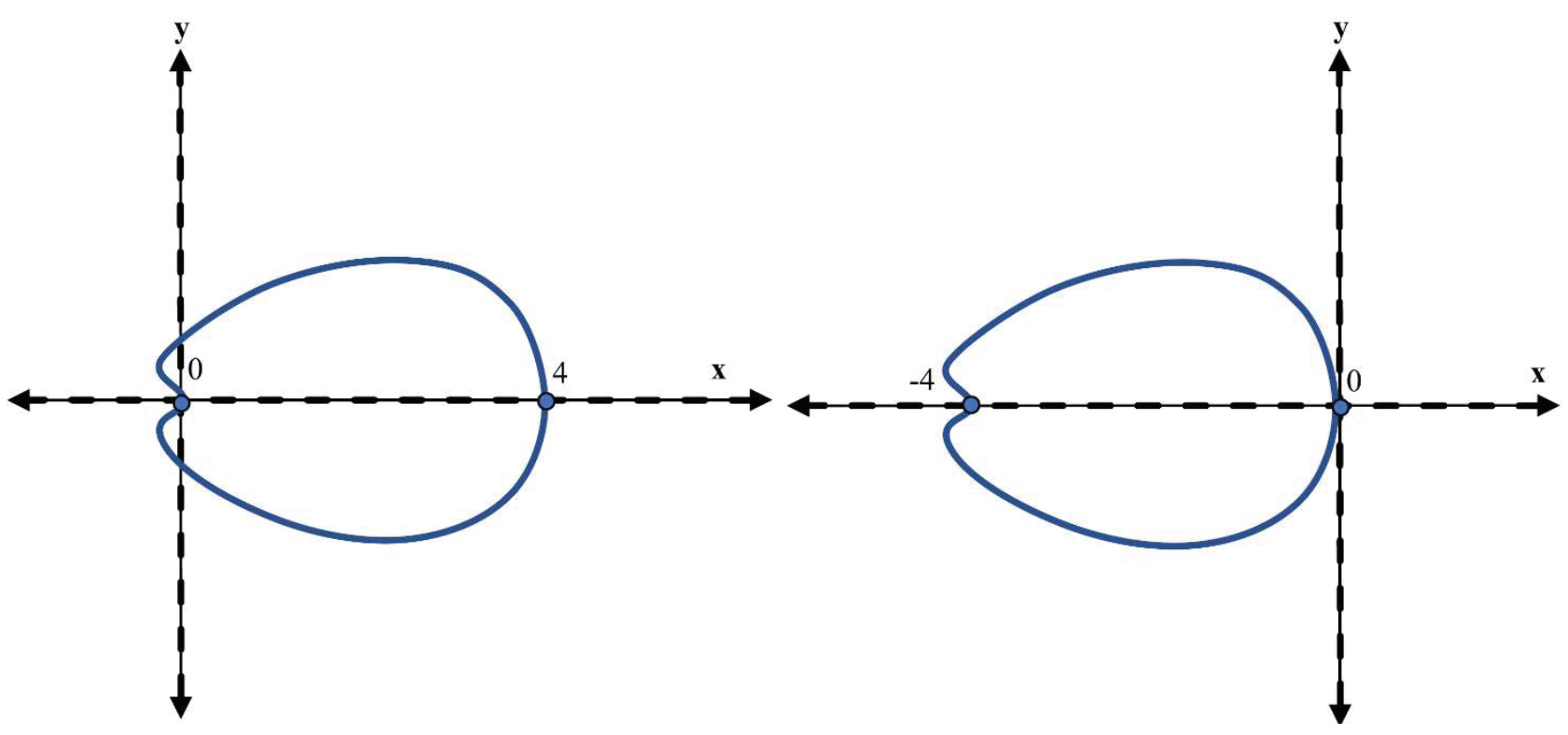

$a$

$b$

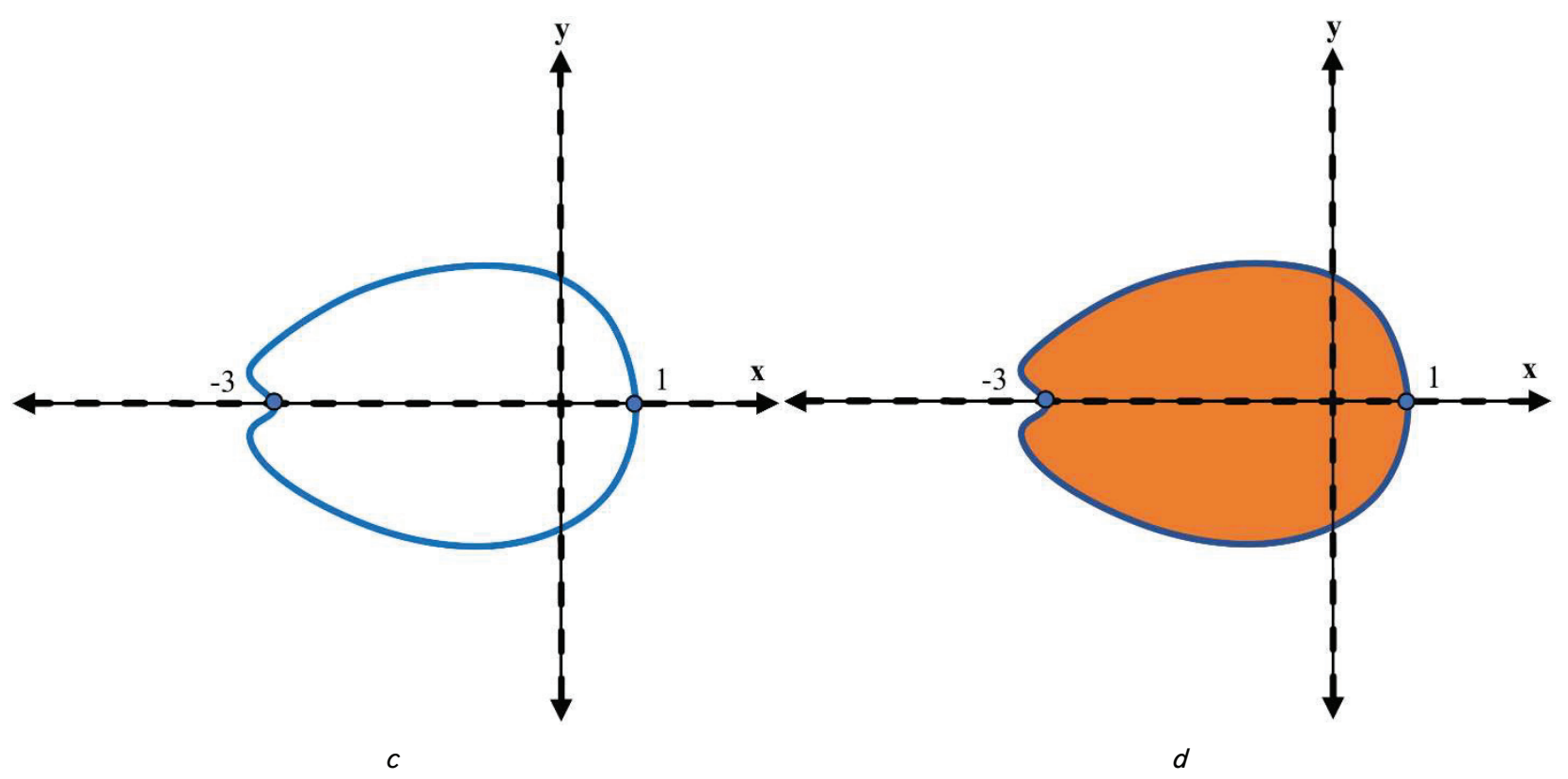

Fig. 5. The representation in the region which contains the function $f^{2}(z, \alpha)$ : $a-\left|1-w^{2}\right|=1 ; b-\left|1-\left(w^{2}-4\right)\right|=1 ; c-\left|5-(\alpha-1)^{2}\right|<1 ; d-\left|5-(\alpha-1)^{2}\right|=1$ 


$$
\left(\alpha^{2}-1\right) z-\alpha^{2}(\alpha+1) z^{2}+2 \alpha^{3} z^{2}-\alpha^{3} z^{4}=0 .
$$

And dividing the equation (3), (4) on $z(z-\alpha-1 / \alpha)$ cancel the effect of fixed points and a function $f(z, \alpha)$, for values $z=0$ and $z=\alpha-1 / \alpha$, let's obtain:

$$
\alpha^{2} z^{2}-\alpha(\alpha+1) z+(\alpha+1)=0 .
$$

And by solving the (10), let's obtain two fixed points for a function $f^{2}(z, \alpha)$

$$
z_{1,2}=\frac{(\alpha+1) \pm \sqrt{\alpha^{2}-z \alpha-3}}{2 \alpha}
$$

The two fixed points $z_{1}$ and $z_{2}$ are attractive if it is $\left|f^{1}\left(z_{1}, \alpha\right) f^{1}\left(z_{2}, \alpha\right)\right|<1$, which will lead to

$$
\left|\alpha^{2}\left(1-2 z_{1}\right) \alpha^{2}\left(1-2 z_{2}\right)\right|<1 .
$$

Compensation for $z_{1}$ and $z_{2}$ in (11), let's obtain

$$
\begin{aligned}
& \left|1-\alpha^{2}+2 \alpha+3\right|<1 \\
& \left|5-(\alpha-1)^{2}\right|<1
\end{aligned}
$$

Let $w$ be a complex number, and suppose that

$$
\begin{aligned}
& w=\alpha-1, \\
& \left|5-w^{2}\right|<1, \\
& \left|1-\left(w^{2}-4\right)\right|<1 .
\end{aligned}
$$

From Theorem (1) $(\beta=1)$, it is found that the shape contains all the points $w$ represent all the points inside the disk circumference equation

$$
\begin{aligned}
& r=2 \cos \theta, \\
& \omega=(2 \cos \theta, \theta), \\
& w^{2}=\left(4 \cos ^{2} \theta, 2 \theta\right),
\end{aligned}
$$

using trigonometric relationship $\theta=1+\cos 2 \theta$, let's obtain

$$
\begin{aligned}
& w^{2}=(2+2 \cos 2 \theta, 2 \theta), \\
& w^{2}=(2+2 \cos \theta, \theta) .
\end{aligned}
$$

In complex plane

$$
\left|1-w^{2}\right|=1
$$

it is cardioid, shown in Fig. 5, refers to (10) then

$$
\left|1-\left(w^{2}-4\right)\right|=1
$$

It is cardioid shown in Fig. 5, b, but

$$
\alpha=w+1,
$$

then

$$
\left|5-(\alpha-1)^{2}\right|=1
$$

it's cardioid shown in Fig. 5, $c$.

\section{Results of analyzing the dynamic behavior of the logistic function}

\section{1. The graphical technique}

Using graphical method the behavior of the logistic map $f(z, \alpha, \beta)=\alpha z(1-z)^{\beta}$ have been investigated to show the relation between the power of the function $\beta$ and the number of humps in the function, let's use the real-time fractal Zoomer Xaos software to represent the function and deduce the positive relationship between the value of the power $\beta$ and the number of humps in function and Fig. 2 demonstrate this relation for the function's iteration and showed the fractals of $f(z)$ for different values of $\beta$.

The iteration of this function inherits the same properties, Fig. 3 represent the fractal of the logistic function iteration, and deduced that as the value of the $\beta$ became big as the number of haps increased, in addition to that the number of humps in a fractal of the first iteration is less than the ones in the second iteration.

\section{2. Fractal of the logistic function iteration}

Fractal of logistic Map was proved to have fixed attractive point, as shown in Theorem 2, the function can be defined by:

$$
\begin{aligned}
& f(f(z))=c\left(c z(1-z)^{1}\right)\left(1-\left(c^{*} z^{*}(1-z)^{1}\right)\right)^{1}= \\
& =\left(-c^{3}\right) z^{4}+\left(2^{*} c^{3}\right) z^{3}+\left(-c^{3}-c^{2}\right) z^{2}+c^{2} z .
\end{aligned}
$$

In addition, this attractive is non zero and belong to the disc $\mid 1-\beta(\alpha-1 \mid<1$. Besides that, The fixed point is attractive when $z=0$, and its path crosse the origin point, As shown in Fig. 4.

\section{3. M Set relationship}

The relationship between the logistic Map and the attractive point was Theoretically investigated considering $M$ set, whereby the logistic $f(z, \alpha, \beta)=z(1-z \beta),(\alpha \in C$ and $\beta \neq 0 \in N)$, has an attractive point belongs to $M=\{\alpha \in C$ and $\beta \in N$ : and the $O\left(Z_{0}\right)$ is bounded under $\left.f(z, \alpha, \beta)\right\}$. However, the set $M$ contains all complex number $\alpha$ in which $|\alpha| \leq(\beta+1)^{((1 / \beta+1))} / \beta$.

\section{4. $f^{2}(z, \alpha)$ function}

The set of all points $\alpha \in C$ in the function $f^{2}(z, \alpha)$ has an attractive fixed point and non-zero belonged to the region shown in Fig. 5 the region is specified as $M=\{\alpha \in C$ and $\beta \in N$ : and the $O\left(Z_{0}\right)$ is bounded under $\left.f(z, \alpha, \beta)\right\}$ and the set $M$ contains all complex number $\alpha$ in which $|\alpha| \leq(\beta+1)((1 / \beta+1)) / \beta$.

\section{Discussion of the research results of logistic function analysis and its behavior}

To analyse the behavior of the logistic function (3), first, let's use the visualization technique to find the relation between the graphical shape of the function and the number of humps that appear from represented the one-dimensional logistic map using the real-time fractal Zoomer Xaos software. The fractal of the first and second iteration in (1) and (2) was tested by the same technique to know the possibilities of knowing more information about these equations.

Fig. 2 demonstrate this relation for the function's iteration and showed that as $\beta$ increased as the number of humps also increase that because of the nature of the logistics function since the power of the function depends on $\beta$ value, i.e. the 
degree of the function became high when the power increases that caused the humps to increase also. The iteration of this logistic function inherits the same properties.

Next, the theoretical technique has been used. Theorem 2 examines the fixed point of fractal of the logistic in (2), In addition, let's determine the area in which this attractive fixed point belongs and the direction of its path. Fig. 4. Showed that the attractive point of the fractal of the logistic in (2) is non-zero and belong to the disc $\mid 1-\beta(\alpha-1 \mid<1$. Besides that, The fixed point is attractive when $z=0$, and its path crosse the origin point.

Theorem 3. The relationship between the logistic Map and the attractive point was theoretically investigated considering the $M$ set. In addition, The set of all points $\alpha \in C$ in the function $f^{2}(z, \alpha)$ in which has a non-zero attractive fixed point was studied to find the relation between $\alpha$ and $\beta$. In theorem 3 let's prove that the logistic $f(z, \alpha, \beta)=z(1-z \beta)$, has an attractive point belongs to $M=\left\{\alpha \in C\right.$ and $\beta \in N$ : and the $O\left(Z_{0}\right)$ is bounded under $f(z, \alpha, \beta)\}$. However, the Set $M$ contains all complex number $\alpha$ in which $|\alpha| \leq(\beta+1)((1 / \beta+1)) / \beta$.

Theorem 4 proved that the set of all points $\alpha \in C$ such that the function $f^{2}(z, \alpha)$ is defined by (8) has fixed points attractive and non-zero and belong to the region specified by shown in Fig. 5. In this figure the region showed that it contains the attractive fixed point of the function $f^{2}(z, \alpha)$ and these points are non-zero, belonged to in the region is specified as $M=\{\alpha \in C$ and $\beta \in N$ : and the $O\left(Z_{0}\right)$ is bounded under $\left.f(z, \alpha, \beta)\right\}$ and the Set $M$ contains all complex number $\alpha$ in which $|\alpha| \leq(\beta+1)((1 / \beta+1)) / \beta$.

Now, let's compare our results of the works in $[27,29]$ and let's find that easy and direct since it depends on visualization technique and gives a quick judge on the function whiteout need to go the derivative direction.

The limitation of the proposed approach is that the when of $\beta$ increases and became greater than 5 .

In this research, we did not study the Logistic Map with Two-Parameters in the complex plane to understand the dynamical behavior of this function.
In future work, we can study the Logistic Map with Two-Parameters in the complex plane and analysis the dynamical behavior of this function using graphical and theoretical techniques, and see how to find the fixed point and attractive point for it.

\section{Conclusions}

1. Using the graphical method, it showed that, the positive relationship between the value of the power $\beta$ and the number of humps in function, such that the number of humps in the graph increases as the power of $(1-z)$ increases. This positive relation is also true for the function's iteration, where the fractals of $f(z)$ have been tested for different values of $\beta$. and it is proven that the iteration of the logistic function inherits the same properties of the original function as shown in Fig. 3. In addition to that, let's manifest the number of humps in the fractal of the first iteration is less than the ones in the second iteration.

2. In the theoretical direction the fixed point of the logistic map and attractive property of the function have been demonstrated, it is proved that the fixed point is non-zero and belongs inside the disc $\mid 1-\beta(\alpha-1 \mid<1$, which crosses the origin point as shown in Fig. 4.

3. Also, the relationship between the logistic map and the attractive point was investigated such that the position of the attractive point lies inside the Mandelbrot set points, and the value of $|\alpha|$ less than $(\beta+1)^{\wedge}((1 / \beta+1)) / \beta$.

4. Using theoretical methods it proven that the function $f^{2}(z, \alpha)$ belong to the region specified by the shape shown in Fig. 5, and carry the same properties of the logistic function i.e. it belongs to the region specified by the set $\mathrm{M}$, and it is possible to study the Logistic Map with Two-Parameters in the complex plane to understand the dynamical behavior of this function.

\section{References}

1. Yu, D., Ta, W., Zhou, Y. (2021). Fractal diffusion patterns of periodic points in the Mandelbrot set. Chaos, Solitons \& Fractals, 153, 111599. doi: https://doi.org/10.1016/j.chaos.2021.111599

2. Schilling, H. (1988). Peitgen, H.-O.; Richter, P. H., The Beauty of Fractals. Images of Complex Dynamical Systems. Berlin etc., Springer-Verlag 1986. XII, 199 pp., 184 figs., many in color, DM 78,-. ISBN 3-540-15851-0. ZAMM - Journal of Applied Mathematics and Mechanics / Zeitschrift Für Angewandte Mathematik Und Mechanik, 68 (10), 512-512. doi: https://doi.org/ 10.1002/zamm.19880681015

3. Brooks, R., Matelski, J. P. (1981). The Dynamics of 2-Generator Subgroups of PSL(2, C). Riemann Surfacese and Related Topics (AM-97), 65-72. doi: https://doi.org/10.1515/9781400881550-007

4. Devaney, R., Keen, L. (Eds). (1989). Chaos and Fractals: The Mathematics Behind the Computer Graphics. Proceedings of Symposia in Applied Mathematics. doi: https://doi.org/10.1090/psapm/039

5. Choudhury, S. R. (1994). Dynamics and Bifurcations (Jack K. Hale and Huseyin Kocak). SIAM Review, 36 (2), 297-299. doi: https://doi.org/10.1137/1036075

6. Liu, S., Pan, Z., Fu, W., Cheng, X. (2017). Fractal generation method based on asymptote family of generalized Mandelbrot set and its application. The Journal of Nonlinear Sciences and Applications, 10 (03), 1148-1161. doi: https://doi.org/10.22436/jnsa.010.03.24

7. May, R. M., Leonard, W. J. (1975). Nonlinear Aspects of Competition Between Three Species. SIAM Journal on Applied Mathematics, 29 (2), 243-253. doi: https://doi.org/10.1137/0129022

8. May, R. M. (1976). Simple mathematical models with very complicated dynamics. Nature, 261 (5560), 459-467. doi: https://doi.org/ $10.1038 / 261459 \mathrm{a} 0$

9. Douady, A., Hubbard, J. H. (2007). Etude' dynamique des polynomes complexes. Societe Mathematique de France. Available at: https://pi.math.cornell.edu/ hubbard/OrsayFrench.pdf

10. Hao, B.-L., Zheng, W.-M. (1998). Applied Symbolic Dynamics and Chaos. World Scientific, 460. doi: https://doi.org/10.1142/3830

11. Introduction (2018). Applied Symbolic Dynamics and Chaos, 1-14. doi: https://doi.org/10.1142/9789813236431_0001 
12. S Chen, S., Feng, S., Fu, W., Zhang, Y. (2021). Logistic Map: Stability and Entrance to Chaos. Journal of Physics: Conference Series, 2014 (1), 012009. doi: https://doi.org/10.1088/1742-6596/2014/1/012009

13. Kwun, Y. C., Tanveer, M., Nazeer, W., Gdawiec, K., Kang, S. M. (2019). Mandelbrot and Julia Sets via Jungck-CR Iteration With s -Convexity. IEEE Access, 7, 12167-12176. doi: https://doi.org/10.1109/access.2019.2892013

14. Mandelbrot, B. B., Wheeler, J. A. (1983). The Fractal Geometry of Nature. American Journal of Physics, 51 (3), 286-287. doi: https://doi.org/10.1119/1.13295

15. Lakhtakia, A., Varadan, V. V., Messier, R., Varadan, V. K. (1987). On the symmetries of the Julia sets for the process z $\Rightarrow$ zp $+c$. Journal of Physics A: Mathematical and General, 20 (11), 3533-3535. doi: https://doi.org/10.1088/0305-4470/20/11/051

16. Kim, T. (2015). Quaternion Julia Set Shape Optimization. Computer Graphics Forum, 34 (5), 167-176. doi: https://doi.org/ $10.1111 /$ cgf.12705

17. Drakopoulos, V., Mimikou, N., Theoharis, T. (2003). An overview of parallel visualisation methods for Mandelbrot and Julia sets. Computers \& Graphics, 27 (4), 635-646. doi: https://doi.org/10.1016/s0097-8493(03)00106-7

18. Sun, Y., Chen, L., Xu, R., Kong, R. (2014). An Image Encryption Algorithm Utilizing Julia Sets and Hilbert Curves. PLoS ONE, 9 (1), e84655. doi: https://doi.org/10.1371/journal.pone.0084655

19. Abbas, S. Q., Abd Almeer, H. A., Ahmed, W. S., Hammid, A. T. (2020). A novel algorithm for generating an edge-regular graph. Procedia Computer Science, 167, 1038-1045. doi: https://doi.org/10.1016/j.procs.2020.03.403

20. Izhikevich, E. M. (2006). Dynamical Systems in Neuroscience. MIT Press. doi: https://doi.org/10.7551/mitpress/2526.001.0001

21. Redona, J. F. (1996). The Mandelbrot set. Theses Digitization Project. Available at: https://scholarworks.lib.csusb.edu/cgi/ viewcontent.cgi? article $=2166 \&$ context $=$ etd-project

22. Fowler, A. C., McGuinness, M. J. (2019). The size of Mandelbrot bulbs. Chaos, Solitons \& Fractals: X, 3, 100019. doi: https://doi.org/ 10.1016/j.csfx.2019.100019

23. Milnor, J., Thurston, W. (1988). On iterated maps of the interval. Lecture Notes in Mathematics, 465-563. doi: https://doi.org/ $10.1007 / \mathrm{bfb} 0082847$

24. Pesin, Y., Climenhaga, V. (2009). Lectures on Fractal Geometry and Dynamical Systems. The Student Mathematical Library. doi: https://doi.org/10.1090/stml/052

25. Kumari, M., Kumari, S., Chugh, R. (2017). International Journal of Mathematics And its Applications Superior Julia Sets and Superior Mandelbrot Sets in SP Orbit. International Journal of Mathematics And its Applications, 5 (2-A), 67-83. Available at: http://ijmaa.in/v5n2-a/67-83.pdf

26. Khamees, M., Ahmed, W. S., Abbas, S. Q. (2020). Train the Multi-Layer Perceptrons Based on Crow Search Algorithm. 2020 1st. Information Technology To Enhance e-Learning and Other Application (IT-ELA). doi: https://doi.org/10.1109/ it-ela50150.2020.9253073

27. Ashish, Cao, J., Chugh, R. (2018). Chaotic behavior of logistic map in superior orbit and an improved chaos-based traffic control model. Nonlinear Dynamics, 94 (2), 959-975. doi: https://doi.org/10.1007/s11071-018-4403-y

28. Kim, Y. I., Feldstein, A. (1997). Bifurcation and k-cycles of a finite-dimensional iterative map, with applications to logistic delay equations. Applied Numerical Mathematics, 24 (2-3), 411-424. doi: https://doi.org/10.1016/s0168-9274(97)00036-6

29. Fruchter, G., Ben-Haim, S. (1991). Stability analysis of one-dimensional dynamical systems applied to an isolated beating heart. Journal of Theoretical Biology, 148 (2), 175-192. doi: https://doi.org/10.1016/s0022-5193(05)80340-6

30. Hirsch, M. W., Smale, S., Devaney, R. L. (2013). Differential Equations, Dynamical Systems, and an Introduction to Chaos. Academic Press. doi: https://doi.org/10.1016/c2009-0-61160-0

31. Weisstein, E. W. Dottie Number.” From MathWorld--A Wolfram Web Resource. Available at: https://mathworld.wolfram.com/ DottieNumber.html

32. Alobaidi, M. H., Idan Kadham, O. (2019). Dynamical Behavior of some families of cubic functions in complex plane. Tikrit Journal of Pure Science, 24 (7), 122. doi: https://doi.org/10.25130/j.v24i7.922

33. Ahmed, W. S. (2013). Construction a MATLAB Program to Solving the Timetable Scheduling Problem. Journal of Engineering and Applied Sciences, 13 (23), 9976-9984. Available at: http://docsdrive.com/pdfs/medwelljournals/jeasci/2018/9976-9984.pdf 